\title{
A study on real-time auditory feedback technique in manipulation task
}

\begin{abstract}
The material presented in this project has illustrated that auditory interfaces are versatile and efficient means of establishing a communication between a computer system and the user. Listening is perhaps the communication channel with the highest bandwidth after visual perception, and certainly a channel whose characteristics are so different from visual perception.

This project had used Microsoft Visual C++ together with OpenGL programming software to create a Tower of Hanoi which has been used as the experiment task. The Tower of Hanoi had been programmed in providing different feedback in real time for the purpose of investigating the effect of auditory feedback to the user. Moreover, the Tower of Hanoi is programmed in stereoscopic for virtual reality manipulation task.

Three types of feedback had been programmed for project investigation. It consists of nonspeech, speech auditory feedback and without feedback. The result of the project showed the participant performance in solving the Tower of Hanoi is better in the non-speech auditory feedback. Beside that, speech auditory feedback provides greatest understanding to the user throughout the experiment, but the drawback is the participant cannot complete the task in shorter time.
\end{abstract}

